\title{
Preferential Attachment in Randomly Grown Networks
}

\author{
Iain S. Weaver* \\ School of Electronics and Computer Science, \\ University of Southampton, University Road, Southampton, SO17 1BJ
}

(Dated: August 20, 2012)

\begin{abstract}
We reintroduce the model of Callaway et al. [1] as a special case of a more general model for random network growth. Vertices are added to the graph at a rate of 1 , while edges are introduced at rate $\delta$. Rather than edges being introduced at random, we allow for a degree of preferential attachment with a linear attachment kernel parametrised by $m$, where the original model is recovered in the limit of no preferential attachment, $m \rightarrow \infty$. As expected, even weak preferential attachment introduces a power-law tail to the degree distribution. Additionally, this generalisation retains a great deal of the tractability of the original along model with a surprising range of behaviour, although key features are modified for finite $m$. In particular, the critical edge density, $\delta_{c}$, which marks the onset of a giant network component is reduced with increasing tendency for preferential attachment and the discontinuity in mean component size exists only for an unbiased random graph. Most notably, the positive degree correlation introduced by the unbiased growth process is diminished, and eventually destroyed by introduction of increasingly skewed degree distributions.
\end{abstract}




\section{INTRODUCTION}

Few networks remain unchanged with time. From the rapidly expanding network of pages that comprise the world wide web, to the power grid, growing alongside our exponentially increasing demand for energy. The timescales associated with changes in these structures can vary from days to decades, while natural systems such as biochemical reaction networks and food webs change on ecological or even evolutionary timescales.

While many of these networks appear mechanistically distinct, the realisation that they may share a number of commonalities has prompted a great deal of research. Of particular interest has been the apparent ubiquity of power-law degree distributions, and what have come to be called scale-free networks. In this regime we find many vertices with few connections, but also a number of extremely well connected nodes, far more than could exist if connectivity were, say, Gaussian. The vast disparity of connectivity suggests there is no characteristic scale, and there is no typical value for the number of connections to a single vertex. Examples include networks of scientific collaborators, where vertices represent authors joined by edges when they collaborate [2] and transport networks such as roads which join at junctions [3]. Further examples of scale-free networks, and power-laws ace discussed in reviews by [4] and [5].

[6] began to answer the question of the origin of these commonalities, demonstrating that scale-free degree distributions may emerge though a process of preferential attachment whereby newly added vertices are connected to existing vertices with probability proportional to their degree. In contrast, [1] introduces a minimal model of network growth in the absence of preferential attachment; vertices are added to a graph at a constant rate and random pairs are connected at a lower rate. Along with a number of interesting mathematical properties, they note that the model history results in older vertices tending to be more highly connected, purely due to having existed longer than younger vertices. Furthermore, these tend to be connected amongst themselves, introducing a positive degree correlation.

We aim to reintroduce the random growth algorithm of [1] as a special instance of a more general algorithm for random growth by allowing either or both ends of added links to attach preferentially to high-degree nodes to varying extent via a linear attachment kernel. Mathematically, this introduces complications, though much of the tractability of the original model is retained. However a number of key observations are disrupted, particularly the 
associative mixing.

The algorithm for growing the network is as follows. At each time step, $t$, we add a single vertex such that the number of vertices is equal to $t$. Additionally, at each time step adds edges are added at the rate $\delta$. While [1] consider only $\delta \leq 1$, this can in principle be very much larger. New edges join a random pair of existing vertices neither of which need be the most recently added vertex. They are joined with probability proportional to their weight which is made up of contributions from their existing connections and a fixed constant. This algorithm continues to $t=t_{\max }$, at which point we analyse the resulting network. The vertex weight is chosen to be the vertex degree $k$ plus a constant $m$, similar to the method implemented by [6]. In our terms, the probability $P_{i, k}$ that vertex $i$ is linked to vertex $j$ by a newly added link is given by

$$
P_{i, j}=\frac{\left(k_{i}+m\right)\left(k_{j}+m\right)}{\sum_{n=1}^{t}\left(k_{n}+m\right)}
$$

where $k$ is the vertex degree, and $m$ parametrises the preference for the new edge to join vertices with a high degree. This model differs significantly from other models of preferential attachment in that networks produced are generally sparse except for high $\delta$ and there is no distinction made between the existing and newly added vertices (as opposed to the fully connected network of [6], where newly added vertices are always connected). We concentrate numerical results on two specific instances of this model, and Fig. 1 shows a section of graphs generated by these different methods.

(a) $m \rightarrow \infty$, where vertices connect randomly, without preference to another vertex exactly as $[1]$.

(b) $m=1$, where both ends of newly added vertices have a strong preference for vertices with several connections.

The analysis which follows is significantly simplified by considering the model case where both ends of a new link have the same for preferential attachment. The extension to allow distinct bias for each end of an edge $\left(m_{1}\right.$ and $\left.m_{2}\right)$ is trivial and displays the same qualitative behaviour. At each stage, we compare analytical results to those derived from numerical simulation. 


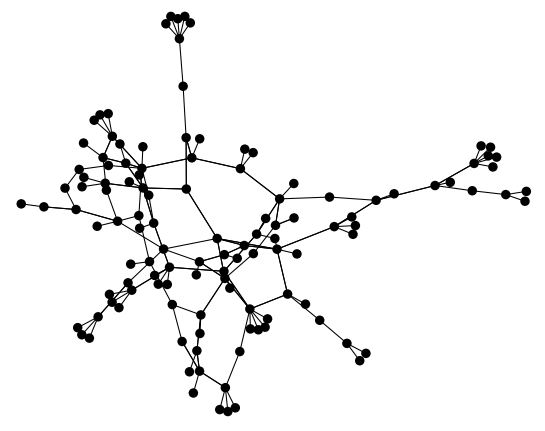

(a) $m \rightarrow \infty$

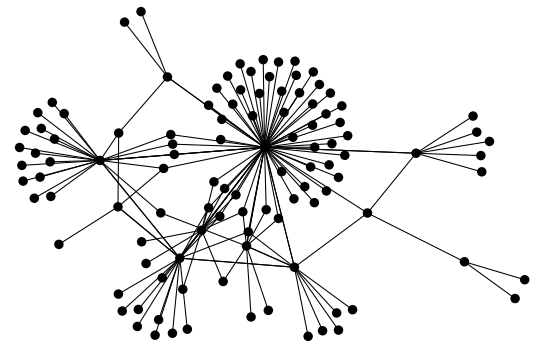

(b) $m=1$

FIG. 1: A sample from the core of a graph grown by our algorithm for the two cases considered with $\delta=\frac{1}{2}$. The images show that finite values for $m$ produces hub and spoke type structures, indicative of power-law degree distributions expected from preferential attachment rules. Singletons are not plotted although graphs generated by such an algorithm are generally very sparse.

\section{DEGREE DISTRIBUTION}

To begin the analysis of this model we follow tradition and derive the steady-state degree distribution for this type of grown graph. The master equation approach gives the expected change in number of vertices with degree $k, d_{k, t}$, between time $t$ and $t+1$. The special case of $d_{0, t}$ is simple since we add isolated vertices at a rate of 1 , and find they are connected at rate

$$
\left\langle d_{0, t+1}-d_{0, t}\right\rangle=1-\frac{2 \delta m}{t(m+2 \delta)} d_{0, t} .
$$

Similarly, the same formulation is applied more generally to higher degree vertices. The change in $d_{k, t}$ is the difference between the expected number of vertices with degree $k-1$ which gain an edge, and those of degree $k$ which gain an edge.

$$
\left\langle d_{k, t+1}-d_{k, t}\right\rangle=\frac{2 \delta(k+m-1)}{t(m+2 \delta)} d_{k-1, t}-\frac{2 \delta(k+m)}{t(m+2 \delta)} d_{k, t} .
$$

From numerical simulation, we find the frequency distribution $d_{k}$ increases linearly with simulation time. As such, we assume the graph grows to a steady state where $d_{k}$ is related to the steady state degree distribution, $p_{k}$, by

$$
d_{k, t}=p_{k} t
$$


This expression can be shown to be appropriately normalised since as stated, model time $t$ is exactly equal to the number of vertices, $\sum_{k} d_{k}$. We seek a solution to eq. (2) of this form by substituting eq. (3) into eq. (2)

$$
p_{k}=\frac{k+m-1}{\frac{m}{2 \delta}+k+m+1} p_{k-1} .
$$

Similarly for eq. (1)

$$
p_{0}=\frac{m+2 \delta}{m+2 \delta m+2 \delta} .
$$

Solving eq. (4) with eq. (5) as a lower bound where $k=1\left(p_{k-1}=p_{0}\right)$ gives

$$
p_{k}=\frac{(m+2 \delta)(m)_{k}}{(m+2 \delta m+2 \delta)\left(\frac{m}{2 \delta}+m+2\right)_{k}}
$$

where we have used Pochhammer notation, defined here as

$$
(m)_{k}=\frac{\Gamma(k+m)}{\Gamma(m)} .
$$

In the limit of $m \rightarrow \infty$, this simplifies significantly.

$$
p_{k}=\frac{(2 \delta)^{k}}{(2 \delta+1)^{k+1}} .
$$

This result gives the degree distribution for case (a) where edges connect random vertices without preference, and is identical to the result for a randomly grown graph [1]. Unsurprisingly, the graph degree distribution has a power-law tail. The exponent can be found in the large $k$ limit using Stirling's approximation. We find

$$
p_{k} \sim k^{-\left(\frac{m}{2 \delta}+2\right)}
$$

except in the case where $m \gg 2 \delta$, where $p_{k}$ tends towards an exponential distribution, illustrated in Fig. 2. Interestingly, strong power-law behaviour $(\gamma<3)$ is only found where $2 \delta>m$. We can say that a highly skewed degree distribution is only found for sufficiently high $\delta$ - the sum weight of vertices due to their edges must be greater than the sum weight of vertices for simply existing in the graph in order to see significant preferential attachment. This effect becomes important later.

\section{GIANT COMPONENT}

We know to expect case (a) to undergo a phase transition across which the expected component size jumps discontinuously [1]. We begin to uncover the corresponding behaviour of case (b) by deriving an expression for the size of the giant connected component, if any. 


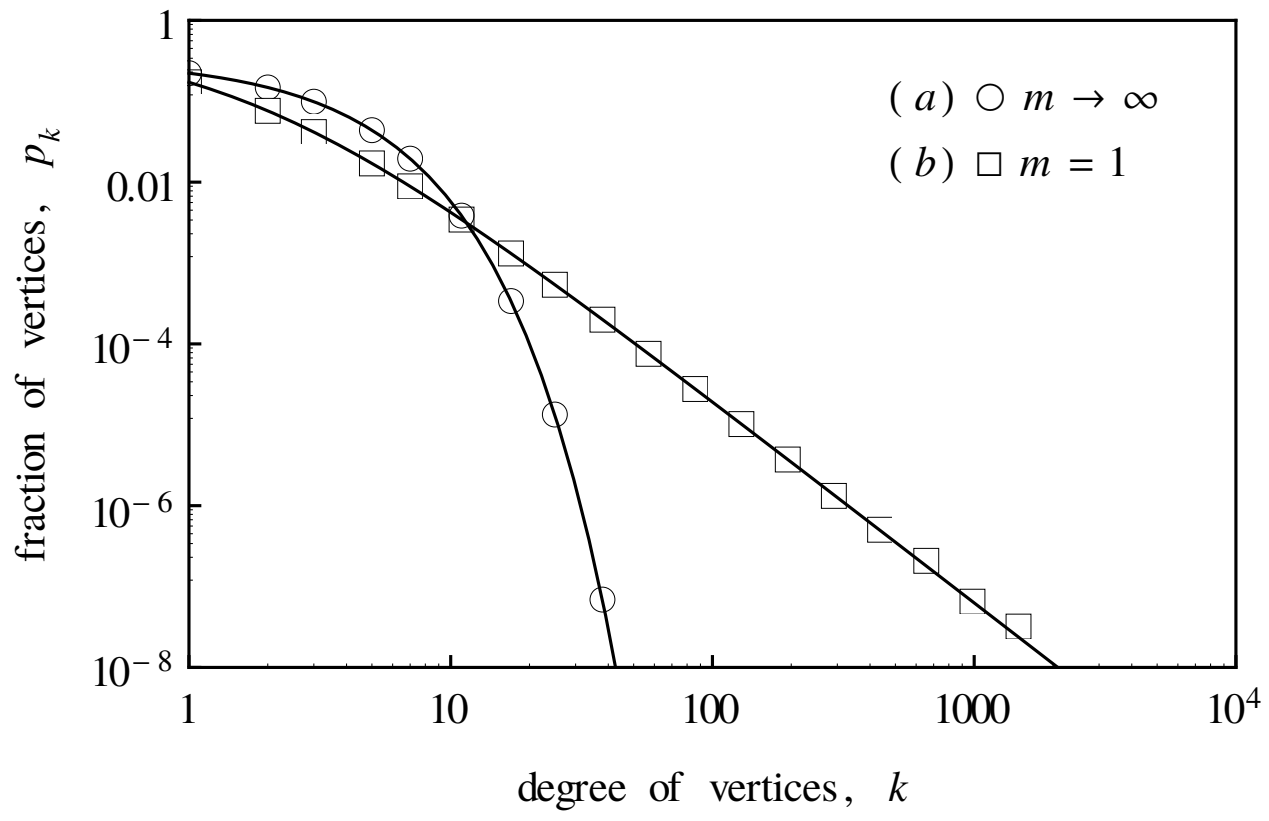

FIG. 2: The degree distributions $p_{k}$ for two model cases considered with $\delta=1$. Plot points indicate distributions are each measured from 50 repetitions of numerical models of $t_{\max }=2 \times 10^{7}$ vertices, while solid lines indicate the numerical results of Eq. (6). Strong agreement between the simulation and analytical progress indicates assumption of large $t$ is satisfactory in this case.

The number of connected components of size $x, N_{x}$ can similarly be derived by a master equation approach. The expected change in the number of connected components of size $x$ at time $t, N_{x, t}$ has two separate contributions. Firstly, from the likelihood of joining different combinations of components which when connected form a component of size $x$, and also from the likelihood of joining a component of size $x$ to any other component. At first, an issue appears to be that we only expect the sum of the weighting within a component to increase linearly with component size for $m \rightarrow \infty$, where individuals are weighted equally. However, by making the assumption that connected components do not contain cycles or self-connections (almost certainly true outside the giant component for large $t$ where the probability of joining vertices from the same component is small) then sum of weights in a finite component of size $x$ is given in Eq. (8), and illustrated in Fig. 3.

$$
\sum_{i=1}^{x}\left(m+k_{i}\right)=(m+2) x-2 .
$$

As before, the smallest components with $x=1$ are introduced at a constant rate of 1 , and 


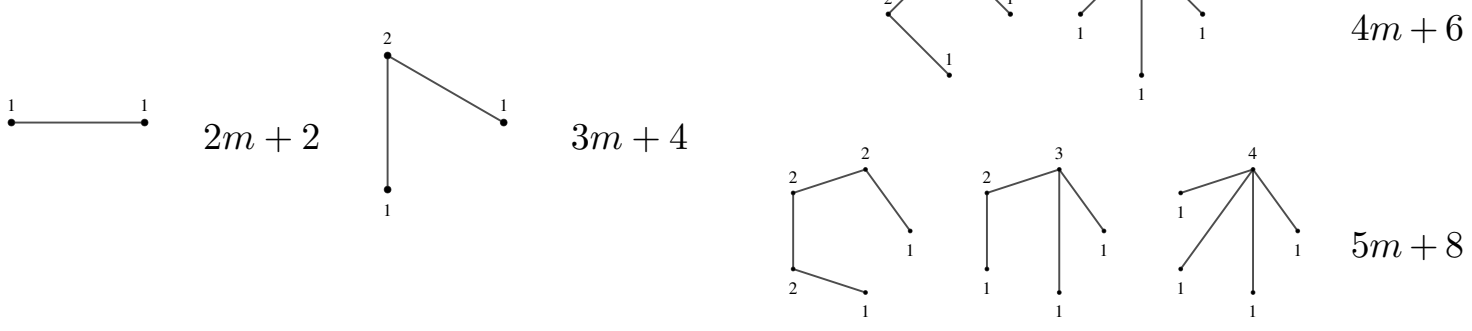

FIG. 3: The weighting of each vertex in a connected component without cycles or self-connections. Since almost all connected components outside the giant component are of this variety, the sum of weights of a component of size $x$ is given, to a good approximation, in Eq. (8).

connected to larger components at a rate of $2 \delta \frac{m}{t(m+2 \delta)} N_{1, t}$.

$$
\left\langle N_{1, t+1}-N_{1, t}\right\rangle=1-\frac{2 \delta m}{t(m+2 \delta)} N_{1, t} .
$$

For $x>1$, we find more generally that

$$
\begin{aligned}
\left\langle N_{x, t+1}-N_{x, t}\right\rangle & =\delta \sum_{y=1}^{x-1} \frac{(m+2) y-2}{t(m+2 \delta)} N_{y, t} \frac{(m+2)(x-y)-2}{t(m+2 \delta)} N_{x-y, t} \\
& -\frac{2 \delta[(m+2) x-2]}{t(m+2 \delta)} N_{x, t} .
\end{aligned}
$$

As before, we seek a solution to Eq. (10) of the form

$$
N_{x, t}=a_{x} t
$$

where $a_{x}$ is the steady state solution to the distribution of component sizes. This can be interpreted as the expected number of connected components of size $x$ per vertex. Substituting this in to Eq. (10)

$$
\begin{aligned}
& a_{k}=-\frac{2 \delta[(m+2) x-2]}{(m+2 \delta)} a_{x} \\
& +\delta \sum_{y=1}^{x-1}\left[\frac{(m+2) y-2}{(m+2 \delta)} a_{y}\right]\left[\frac{(m+2)(x-y)-2}{(m+2 \delta)} a_{x-y}\right] .
\end{aligned}
$$

Similarly for Eq. (9)

$$
a_{1}=\frac{m+2 \delta}{m+2 \delta m+2 \delta} .
$$


This can be solved iteratively to find the fraction of the network connected into the giant component, though we seek a more useful formulation through the use of generating functions. We define the generating function for the probability that a randomly chosen vertex belongs to a finite connected cluster of size $x, g(z)$ as

$$
g(z)=\sum_{x} a_{x} e^{x z}
$$

Recall that the distribution $a_{k}$ is not normalised. The sum $g(0)$ gives the ratio of the number of connected components to the number of vertices, such that $g(0)=1$ only when $\delta=0$. We are interested in $g(0)$ along with its first derivative,

$$
g^{\prime}(z)=\sum_{x} x a_{x} e^{x z}
$$

The interpretation of $x a_{x}$ is the likelihood of a randomly selected vertex belonging to a connected component of size $k$. The sum $g^{\prime}(0)$ is therefore unity when no giant connected component exists (all components have finite size). Above the phase transition, the fraction of the graph occupied by the giant component, $S$, can be extracted by

$$
S=1-g^{\prime}(0)
$$

Multiplying Eq. (12) by $e^{x z}$, summing over $x$ and substituting Eq. (12) for the case where $x=1$ gives

$$
\begin{aligned}
& 2 \delta(m+2 \delta)(m+2) g^{\prime}(z)= \\
& \delta(m+2)^{2} g^{\prime}(z)^{2}+4 \delta g(z)^{2}-4 \delta(m+2) g(z) g^{\prime}(z) \\
& -(m+2 \delta)(m-2 \delta) g(z)+(m+2 \delta)^{2} .
\end{aligned}
$$

Solving this equation with the initial condition $\left(z_{0}, g\left(z_{0}\right)\right)=\left(e^{z_{0}}, e^{z_{0}} a_{1}\right)$ for large negative values of $z_{0}$. It is found that $\ln 10^{-6}$ provides sufficient accuracy. The giant component size is shown in Fig. 4.

As we might expect, the strong preference for vertices of higher degree in case (b) causes a giant connected component to emerge at smaller values of $\delta$ compared with the random model (a). The reason for this is that the limited edges will tend to be concentrated over a smaller fraction of vertices, producing a giant connected component. However, its growth is inhibited by an increasing number of internal connections which do not increase its absolute size. 


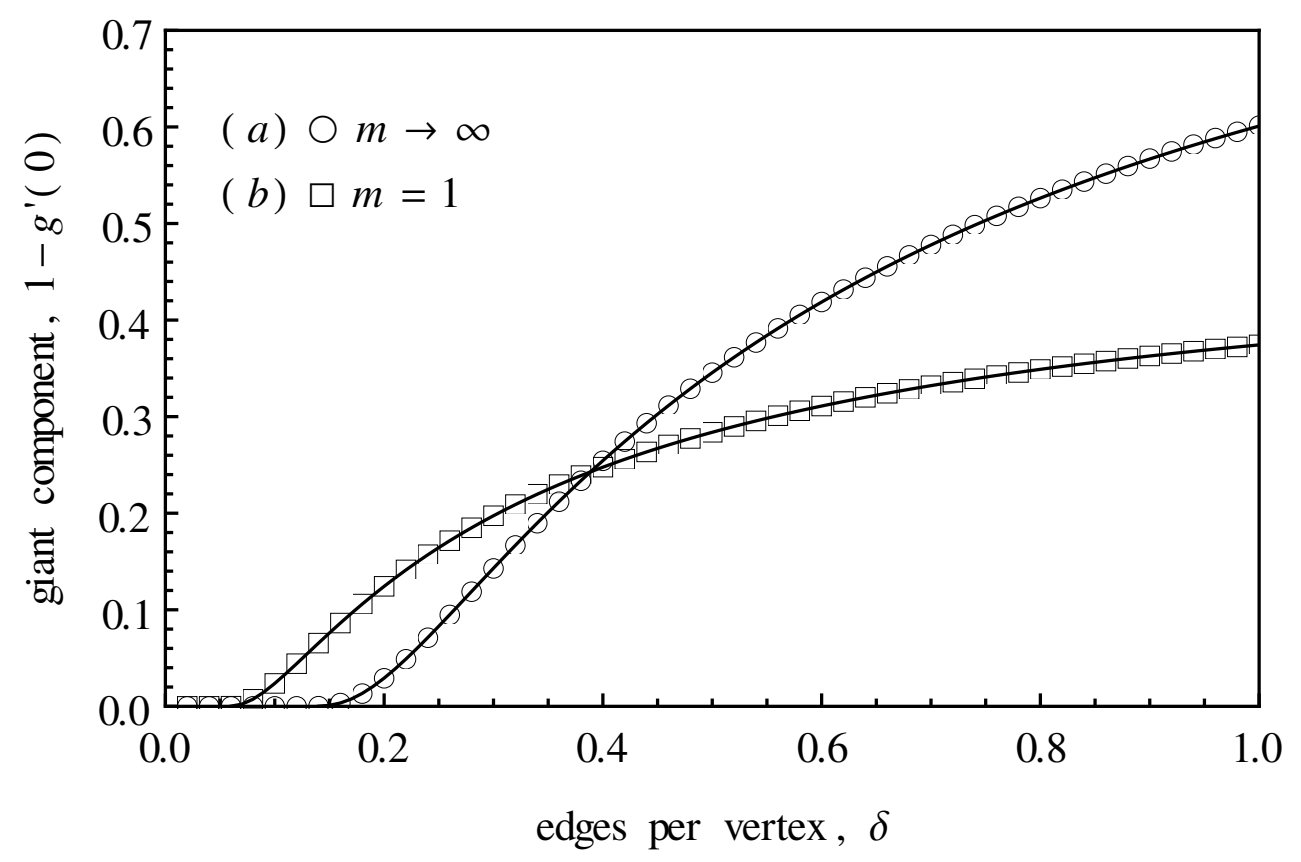

FIG. 4: The fraction of the network connected to the giant component for the two model cases. Plot points are each measured from 25 repetitions of numerical models of $t_{\max }=20 \times 10^{6}$ vertices, while solid lines indicate the results of Eq. (13) with numerical integration of Eq. (14).

\section{MEAN COMPONENT SIZE}

We can find exactly where the giant component emerges by, in addition to $g^{\prime}(0)$, deriving $g^{\prime \prime}(0)$ defined as

$$
g^{\prime \prime}(z)=\sum_{x=1}^{\infty} x^{2} a_{x} e^{x z} .
$$

This is a useful property since it is related to the expected component size $\langle s\rangle$ by

$$
\langle s\rangle=\frac{\sum_{x=1}^{\infty} x^{2} a_{x}}{\sum_{x=1}^{\infty} x a_{x}}=\frac{g^{\prime \prime}(0)}{g^{\prime}(0)} .
$$

The position of the phase transition can be determined by examining the form of $g(0)$ in the $g^{\prime}(0)=1$ regime, that is to say the range of $\delta$ for which no giant connected component exists. Substituting this into Eq. (14) at $z=0$, and solving for $g(0)$ gives

$$
g(0)=\frac{m^{2}-4 \delta^{2}+4 \delta(m+2) \pm(m+2 \delta)^{2}}{8 d} .
$$

Since we know that as $\delta \rightarrow 0$ the ratio $g(0)$ approaches unity, we choose the negative signed solution, which contracts Eq. (15) to $g(0)=1-\delta$. This exactly reflects our previous 
assumption that finite components contain no internal connections, and new edges always join components. Differentiating Eq. (14) and applying L'Hôpital's rule in the limit $z \rightarrow 0$ with $g(0)=1-\delta$ and $g^{\prime}(0)=1$ gives

$$
\begin{aligned}
g^{\prime \prime}(0) & =\frac{m^{2}+4 \delta(3 m+\delta+4)}{4 \delta(m+2)^{2}} \\
& \pm \frac{(2 \delta+m) \sqrt{m^{2}+4 \delta^{2}-4 \delta m(2 m+3)}}{4 \delta(m+2)^{2}}
\end{aligned}
$$

and we define $\delta_{c}$ as

$$
\delta_{c}=\frac{1}{2}\left(3 m+2 m^{2}-2 \sqrt{2 m^{2}+3 m^{3}+m^{4}}\right),
$$

noting that Eq. (16) is only valid for $\delta \leq \delta_{c}$. We choose the solution with the negative sign since we know that for $\delta=0$, each vertex belongs to a component of size one, such that $\langle s\rangle=1 . \delta_{c}$ indicates the position of the emergence of a giant connected component. Expanding this in the limit $m \rightarrow \infty$ gives $\delta_{c}=\frac{1}{8}$, exactly as with the random grown graph [1]. In the absence of any closed form expression of $g(0)$ from Eq. (14) for the general case of $g^{\prime}(0)<1$, we solve for $g^{\prime \prime}(0)$ numerically with the initial condition $\left(z_{0}, g\left(z_{0}\right), g^{\prime}\left(z_{0}\right)\right)=$ $\left(e^{z_{0}}, e^{z_{0}} a_{1}, e^{z_{0}} a_{1}\right)$ for large negative values of $z_{0}$. Results are displayed in Fig. 5.

Interestingly, it appears as though a discontinuity at $\delta_{c}$ only exists for the randomly grown graph shown in Fig. 5a, where $g^{\prime \prime}(0)$ and therefore $\langle s\rangle$ jumps from 2 to 4 at $\delta_{c}=\frac{1}{8}$. The presence of preferential attachment breaks this, and numerical integration of Eq. (14) suggests a smooth change in $\langle s\rangle$ across the transition. Additionally, $\delta_{c}$ is shown to shift left for small $m$, as indicated by Fig. 4 .

\section{DEGREE CORRELATION}

We have found an aspect in which the randomly grown graph represents a special case of a more general grown graph with qualitatively different properties. This can also be seen by examination of associative mixing in our model $[1,6,7]$. The explanation for positive associative mixing in the randomly grown graph is that older vertices, introduced at small $t$ will not only accumulate more connections, but they will be more likely to find connections amongst themselves in comparison to younger vertices introduced at larger $t$. The result is that vertices of high degree are likely to attract connections to other vertices of high degree, resulting in associative mixing. 


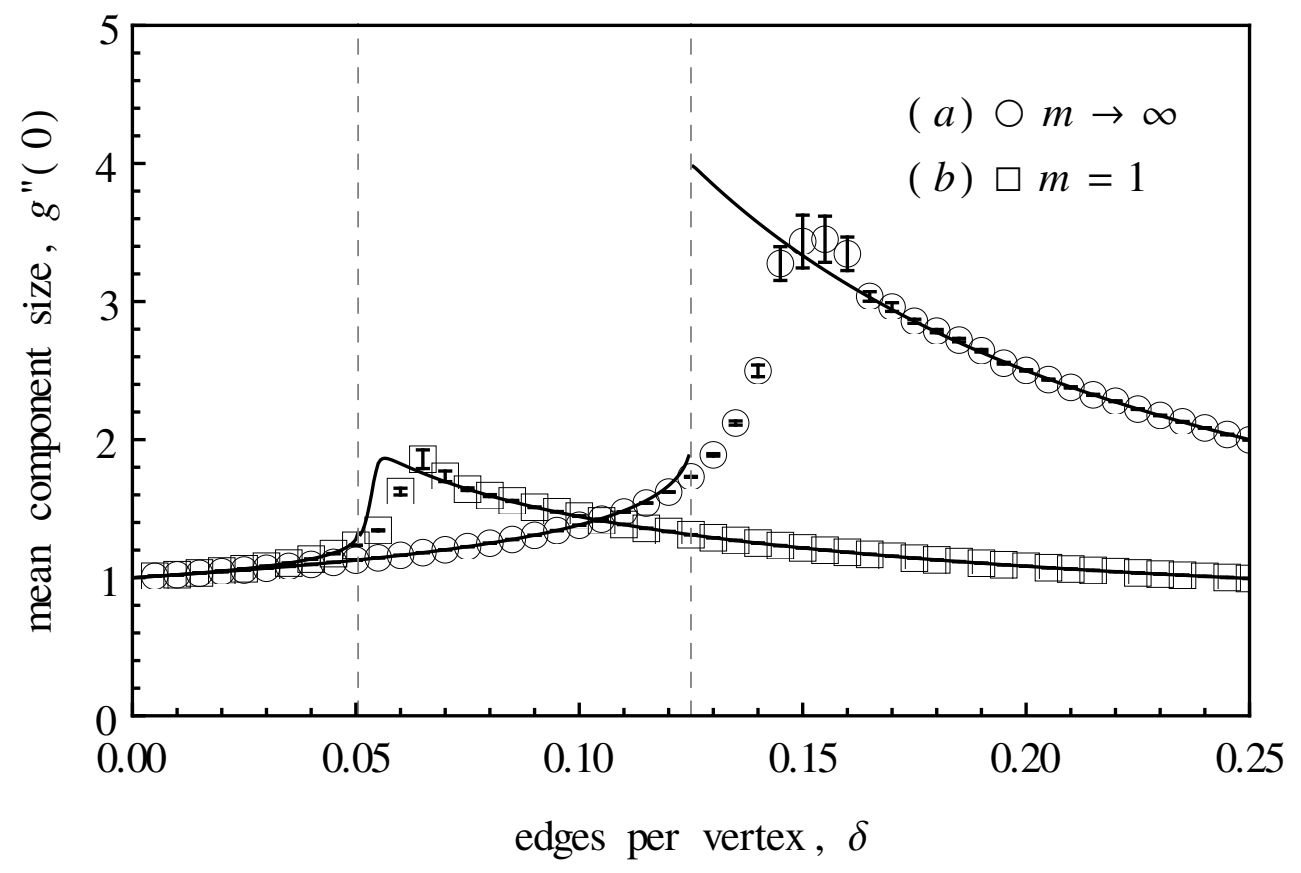

(a) $m \rightarrow \infty$

FIG. 5: Plot points are each measured from 25 repetitions of numerical models of $t_{\max }=20 \times 10^{6}$ vertices, while solid lines indicate the results of numerical integration of Eq. (14). Dashed lines indicate the position of the emergence of a giant component from Eq. (17).

The number of edges connecting vertices with degree $k$ to $l$ at time $t$, is defined as $E_{k l, t}$ (as introduced by [8]). This term has three contributions;

1. $E_{k l, t}$ is increased when a vertex of degree $k-1$ is already connected to a vertex of degree $l$ and receives another connection.

2. $E_{k l, t}$ is increased by unconnected vertices of degree $k-1$ and $l-1$ becoming connected

3. $E_{k l, t}$ is decreased when vertices of degree $k$ and $l$ are connected, and either receives an additional connection 
The master equation resulting from these terms is

$$
\begin{aligned}
& \left\langle E_{k l, t+1}-E_{k l, t}\right\rangle= \\
& 2 \delta\left[\frac{d_{k-1, t}(k+m-1)}{(2 \delta+m) t} \frac{E_{k-1, l, t}}{d_{k-1, t}}+\frac{d_{l-1, t}(l+m-1)}{(2 \delta+m) t} \frac{E_{k, l-1, t}}{d_{l-1, t}}\right] \\
& +2 \delta\left[\frac{k+m-1}{(2 \delta+m) t} d_{k-1, t} \frac{l+m-1}{(2 \delta+m) t} d_{l-1, t}\right] \\
& -2 \delta\left[\frac{d_{k, t}(k+m)}{(2 \delta+m) t} \frac{E_{k, l, t}}{d_{k, t}}+\frac{d_{l, t}(l+m)}{(2 \delta+m) t} \frac{E_{k, l, t}}{d_{l, t}}\right] .
\end{aligned}
$$

By adding that $E_{0, l, t}=E_{k, 0, t}=0$ for all $k$ and $l$ (vertices with no connections cannot have neighbours), Eq. (18) is general enough to encompass $E_{1,1, t}$, requiring no separate definition as found previously. In the steady state, we assume

$$
E_{k l, t}=2 \delta t e_{k l}
$$

The matrix $e_{k l}$ gives the distribution of the degree of vertices at each end of a randomly chosen edge, normalised such that $\sum_{k} e_{k l}=p_{k}$. The factor of 2 comes from the fact that $E_{k l, t}$ is symmetrical under the interchange of $l$ and $k$, and summation over $k$ and $l$ will double count the total number of edges, $\delta t$.

Substituting this into Eq. (18), then solving for $e_{k l}$ gives

$$
\begin{aligned}
e_{k l}= & \frac{2 \delta\left[(k+m-1) e_{k-1, l}+(l+m-1) e_{k, l-1}\right]}{m+2 \delta(k+l+2 m+1)} \\
& +\frac{(k+m-1)(l+m-1) p_{k-1} p_{l-1}}{(2 \delta+m)[m+2 \delta(k+l+2 m+1)]} .
\end{aligned}
$$

We translate this matrix into the degree correlation coefficient, $\rho$, following [1].

$$
\rho=\frac{c}{\sigma^{2}}
$$

where $c$ and $\sigma^{2}$ are defined as

$$
\sigma^{2}=\frac{\sum_{k}(k-\mu)^{2} k p_{k}}{\sum_{k} k p_{k}} c=\sum_{k l}(k-\mu)(l-\mu) e_{k l}
$$

the variance of the vertex degree distribution at either end of a random edge, and the covariance of the degrees of vertices at the ends of a random edge respectively. In both cases $\mu$ is the mean degree of a vertex at the end of a randomly chosen edge

$$
\mu=\frac{\sum_{k} k^{2} p_{k}}{\sum_{k} k p_{k}}
$$

Here, the skewed degree distribution has an impact. Examining the long tail of the degree distribution given in Eq. (7) we can see that the variance of the distribution diverges when 


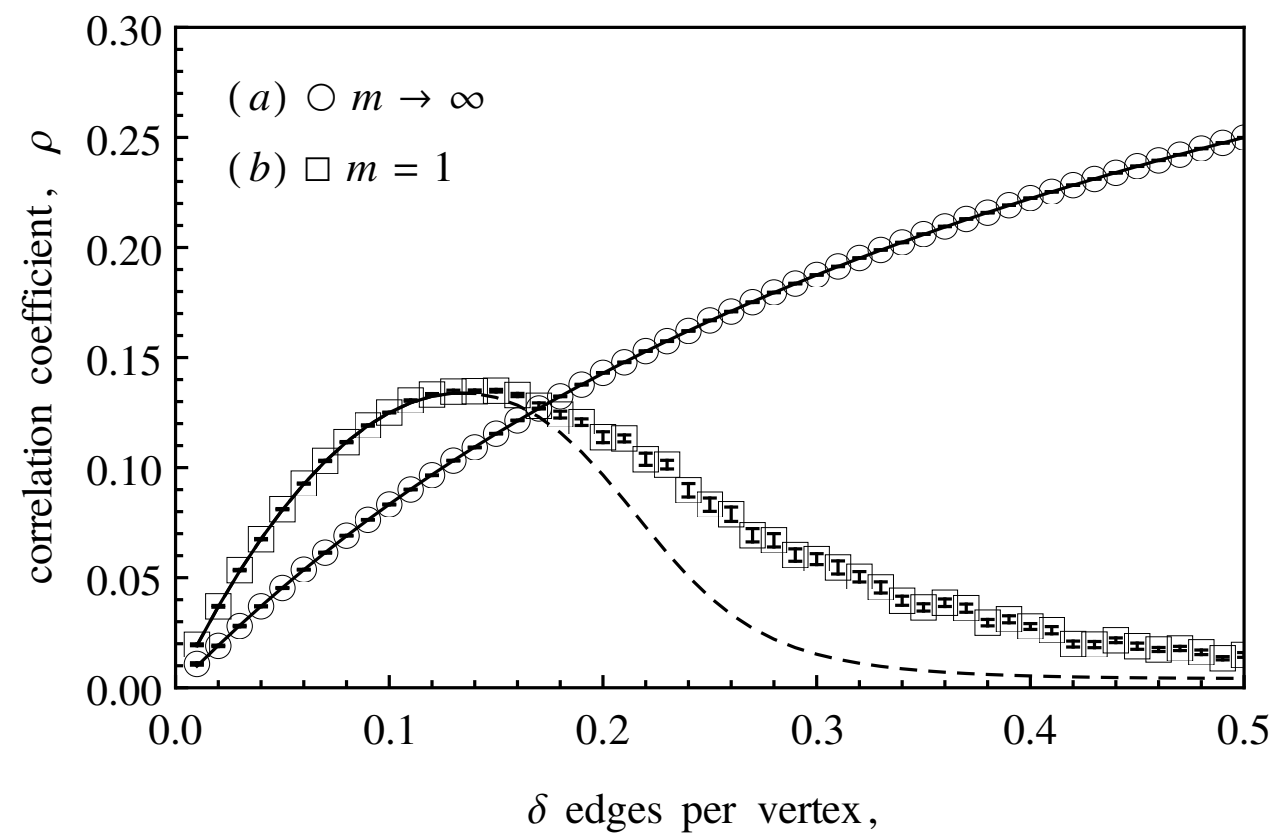

FIG. 6: The correlation coefficient $\rho$ for the two model cases considered. Plot points are each measured from 25 repetitions of numerical models of $t_{\max }=20 \times 10^{6}$ vertices and $\rho$ is calculated using Eq. (21), while solid lines indicate the results of numerical iteration of $10^{5}$ terms of Eq. (19), from which $\rho$ is calculated using Eq. (20).

the exponent of the tail $\frac{m}{2 \delta}+2 \leq 3$. Here $\mu$ diverges and the degree correlation is exactly zero.

Fig. 6 shows the results of numerical iteration of Eq. (20) with Eq. (19), compared to numerical simulation, where the degree correlation is found through explicit enumeration of edges in the simulation [7]

$$
\rho=\frac{M^{-1} \sum_{i} j_{i} k_{i}-\left[M^{-1} \sum_{i} \frac{1}{2}\left(j_{i}+k_{i}\right)\right]^{2}}{M^{-1} \sum_{i} \frac{1}{2}\left(j_{i}^{2}+k_{i}^{2}\right)-\left[M^{-1} \sum_{i} \frac{1}{2}\left(j_{i}+k_{i}\right)\right]^{2}} .
$$

The problem encountered here is two-fold. Firstly, in the strong power-law regime, we find a highly skewed degree distribution predicted by Eq. (6) for small values of $m$. At this stage, numerical iteration of Eq. (19) requires a large number of terms to be considered for good accuracy. In addition to this, we suspect that even for $t_{\max }=20 \times 10^{6}$ vertices, the effects of the finite model size are highly significant. Fig. 7 illustrates the finite size effects; the rate of convergence of $\rho$ with large $t_{\max }$ is slow, and dominated by rare, highly connected vertices. 


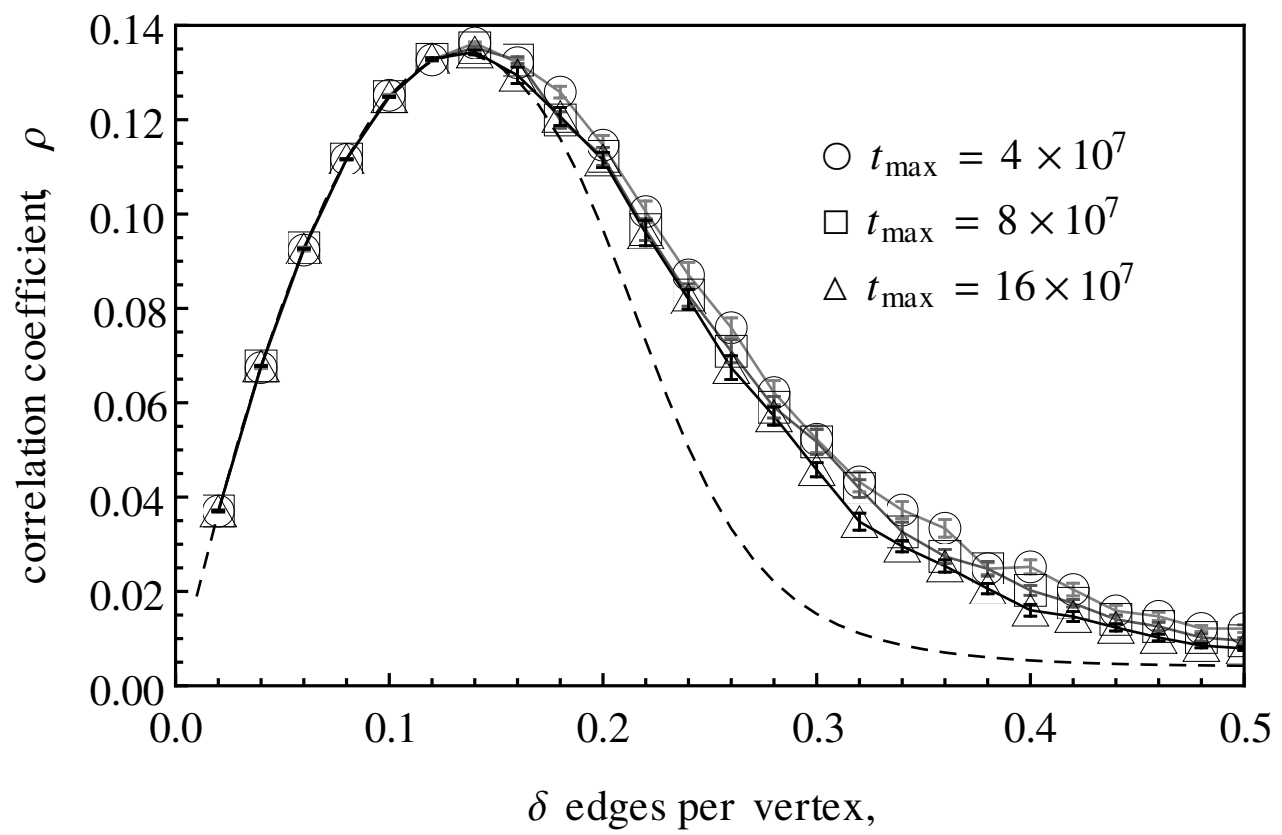

FIG. 7: The correlation coefficient $\rho$ for a range of model sizes. Points show the results of 10 repetitions of models run to $t_{\max }$, while the dashed line indicates numerical iteration of $10^{5}$ terms of eq. (19).

Fig. 6 shows a surprising contrast between the networks grown with some tendency for preferential attachment and the randomly grown case with $m \rightarrow \infty$. While all instances have a historical tendency for older vertices both to have higher connectivity and be more likely to be connected, we find that this does not imply $\rho>0$. For any finite $m, \rho$ increases to a maximum at $\delta=\delta_{0}$, where $\delta_{0}$ is some increasing function of $m$, before decaying with increasing $\delta$ Although we find that finite size allows the model to maintain a positive correlation even for highly skewed degree distributions. At the point $m=2 \delta$ the variance in degree distribution diverges, and degree correlation is exactly zero. The limit of this is the case of unbiased growth $m \rightarrow \infty$ where $\delta_{0} \rightarrow \infty$, and positive associative mixing approaches its maxima asymptotically.

\section{CONCLUSION}

We have introduced a general form of a randomly grown graph [1] to allow for one, or both ends of new edges to connect with a bias towards to high degree vertices. Qualitatively similar properties are found in the case where both ends have the same propensity for 
preferential connection. This extension retains a great deal of the tractability of the special case of [1] and reveals that a number of properties observed are unique to the case of zero preferential attachment.

First, we have shown that addition of preferential attachment in randomly grown networks introduces skewed degree distributions with power-law tails. We find that even for weak preferential attachment the discontinuity in the mean component size with increasing $\delta$ vanishes. In its place we find a sharp but continuous change in mean component size across the phase transition at $\delta_{c}$ which marks the appearance of a giant connected component. The position of $\delta_{c}$ decreases with stronger preferential attachment as would be expected; the introduction of preferential attachment encourages the existence of a giant component at small $\delta$ compared to the unbiased case by concentrating edges between older well-connected vertices. However, the onset of the giant component does not correspond to a maxima in mean component sizes as with the case of unbiased network growth.

Moreover, the addition of preferential attachment finds degree correlation to increase up to some critical value $\delta_{0}$ before decaying to zero for increasingly well connected graphs, with increasingly skewed degree distributions. This is opposed to the asymptotically increasing degree correlation found from unbiased random growth. Even large simulations indicate this convergence to be slow, as the behaviour is dominated by rare, highly connected vertices indicative of scale-free networks. A similar result is obtained by [9] where scale-free networks are shown to be able to possess associative mixing to some extent. This result is particularly surprising as the positive correlation in the random case is presumed to be a result of the history embedded in the network growth. However, we have shown that while history plays a part it does not imply we will find associative mixing for networks with strongly skewed degree distributions.

This work was supported by an EPSRC Doctoral Training Centre grant (EP/G03690X/1).

* isw1g10@soton.ac.uk; http://cmg.soton.ac.uk/people/isw1g10/; With thanks to Dr. Adam Prügel-Bennett for his time and patient discussion

[1] D. S. Callaway, J. E. Hopcroft, J. M. Kleinberg, M. E. J. Newman, and S. H. Strogatz, “doibase-10.1103/PhysRevE.64.041902 Phys. Rev. E 64 (2001), 10.1103/PhysRevE.64.041902. 
[2] M. Newman, Proceedings of the National Academy of Sciences 98, 404 (2001).

[3] V. Kalapala, V. Sanwalani, A. Clauset, and C. Moore, Fhttp://dx.doi.org/10.1103/PhysRevE.73.026130 Phys. Rev. E 73, 026130 (2006).

[4] M. Mitzenmacher, Internet mathematics 1, 226 (2004).

[5] M. Newman, Contemporary physics 46, 323 (2005).

[6] A. L. Barabasi and R. Albert, "doibase-10.1126/science.286.5439.509 ArXiv Condensed Matter e-prints (1999), 10.1126/science.286.5439.509, - -http://arxiv.org/abs/arXiv:cond-mat/9910332 arXiv:cond-mat/9910332 .

[7] M. E. J. Newman, "doibase-10.1103/PhysRevLett.89.208701 Phys. Rev. Lett. 89, 208701 (2002).

[8] P. L. Krapivsky, G. J. Rodgers, and S. Redner, "doibase-10.1103/PhysRevLett.86.5401 Phys. Rev. Lett. 86, 5401 (2001).

[9] P. L. Krapivsky and S. Redner, "doibase-10.1103/PhysRevE.63.066123 Phys. Rev. E 63, 066123 (2001). 